\title{
Structural conservation and vernacular construction
}

\author{
CHAPTER · SEPTEMBER 2015
}

DOI: $10.1201 /$ b18856-8

READS

58

4 AUTHORS:

Paulo B. Lourenco

University of Minho

578 PUBLICATIONS $\quad 4,027$ CITATIONS

SEE PROFILE

Graça Vasconcelos

University of Minho

104 PUBLICATIONS 349 CITATIONS

SEE PROFILE
H. Varum

University of Porto

413 PUBLICATIONS 1,012 CITATIONS

SEE PROFILE

Hugo Rodrigues

Instituto Politécnico de Leiria

131 PUBLICATIONS 376 CITATIONS

SEE PROFILE 


\title{
Structural conservation and vernacular construction
}

\author{
P.B. Lourenço \\ University of Minho, Guimarães, Portugal \\ H. Varum \\ University of Porto, Porto, Portugal \\ G. Vasconcelos \\ University of Minho, Guimarães, Portugal
}

H. Rodrigues

Polytechnic Institute of Leiria, Leiria, Portugal

\begin{abstract}
Modern societies understand built cultural heritage, including vernacular construction, as a landmark of culture and diversity, which needs to be protected and brought to the next generations in suitable condition. Still, a large part of this heritage is affected by structural problems that menace the safety of buildings and people. In the case of vernacular construction, deterioration due to abandonment is often present, making the phenomena of urbanization one of the most important menaces to this built heritage. The developments in the areas of inspection, non-destructive testing, monitoring and structural analysis of historical constructions, together with recent guidelines for reuse and conservation, allow for safer, economical and adequate remedial measures, as discussed in this paper.
\end{abstract}

\section{INTRODUCTION}

\subsection{Cultural heritage and risk reduction}

Cultural heritage buildings are particularly vulnerable to disasters because they are often deteriorated and damaged, because they were built with materials with low resistance, and because they are heavy and the connections between the various structural components are often insufficient. The main causes for damage are the lack of maintenance and waterinduced deterioration (from rain or rising damp), soil settlements and extreme events such as earthquakes, see Fig. 1. Extreme events often lead to disasters, in light of the high vulnerability, e.g. Neves et al. (2012) and Leite et at. (2013). Still, there are many other causes of damage, namely: high stresses due to gravity loading, alterations in lay-out or construction, cyclic environmental actions, climate change, physical attack from wind and water, chemical and biological attack, vegetation growth, fire, floods, vibration and micro-tremors, and anthropogenic actions.

The built cultural heritage includes archaeological remains, monuments, dwellings and vernacular buildings, groups of buildings, ancient city centers, and historical urban texture but also outstanding engineering works from antiquity to present, industrial heritage, $20^{\text {th }}$ century heritage in steel or reinforced concrete and even modern heritage. Despite the extension of cultural heritage legislation and protection to groups of buildings and urban spaces, and despite the listing (inventory) of complete town centers, the instruments and the application of monument protec- tion is still fundamentally 'object' centered. The approach for risk reduction targeted to groups of buildings, urban spaces and isolated buildings is known, being necessary to: (i) characterize the existing built heritage; (ii) perform simplified analysis at the territorial level to estimate the vulnerability and risk of this heritage; (iii) in cases that are identified with higher risk in the previous step, perform detailed analyses to confirm the vulnerability and risk; (iv) define a plan with long-term intervention measures and their costs, taking into account the observed risk; (v) implement the plan, with periodic reviews of time and costs, considering the economic constraints and the costs incurred in the interventions. Such a strategy requires political and societal commitment to become reality.

\subsection{Masonry and timber}

Most of the existing built heritage, particularly in the case of vernacular construction, is made with the socalled traditional materials (masonry, including earth, and timber). In many cases of vernacular construction, structural walls are made of masonry, while floors and roofs are made of timber. In some cases, structural walls are also made of halftimbered construction. The influence factors on construction practice were mainly the local culture and wealth, the knowledge of materials and tools, the availability of material and aesthetic reasons. Ancient buildings are frequently characterized by their durability, which enabled them to remain in a good condition throughout relatively long time periods. 


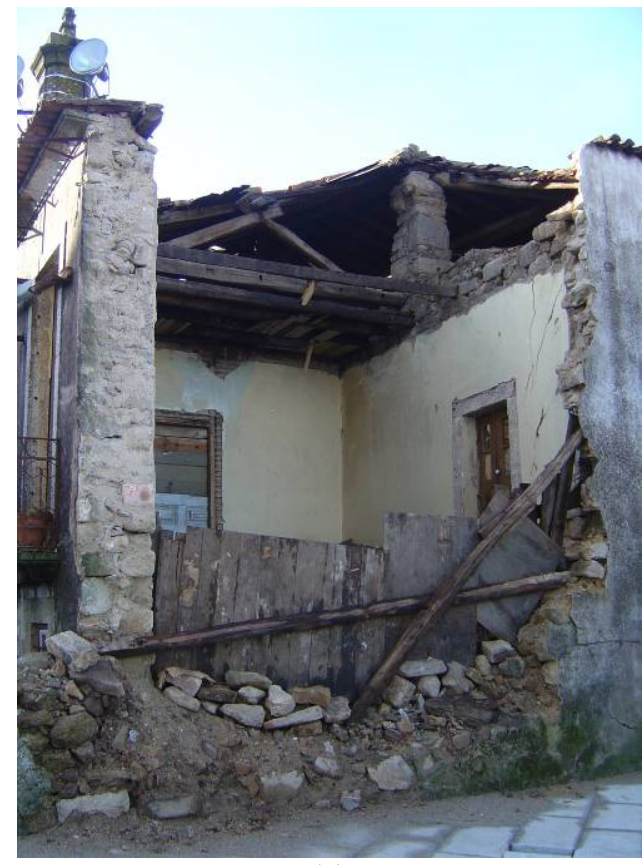

(a)

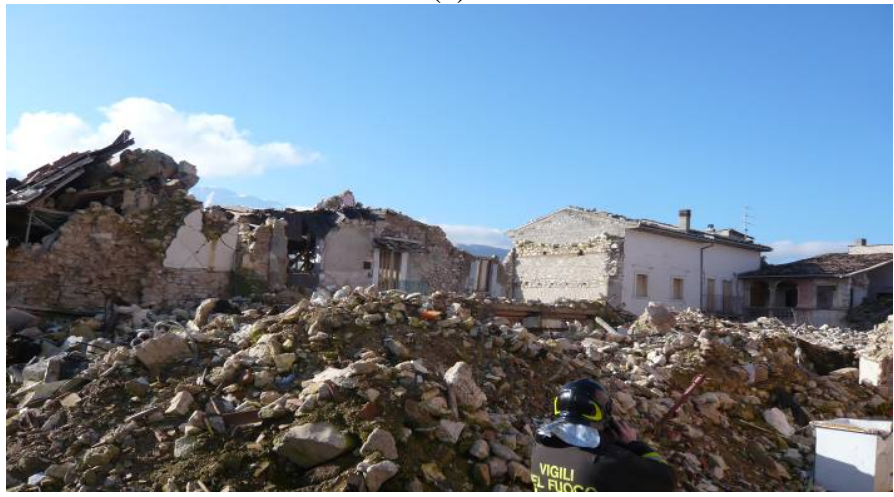

(b)

Figure 1. Collapse of vernacular construction: (a) Progressive damage due to water leakage from roof; (b) L'Aquila earthquake, 2009.

Innumerable variations of masonry materials, techniques and applications occurred during the course of time. The first masonry material to be used was probably stone. In addition to the use of stone also mud brick started to be used as a masonry material, as it could be easily produced. Brick was lighter than stone, easy to mold and formed a wall that was fire resistant and durable. The practice of burning brick probably started with the observation that the brick was stronger and more durable. Another component of masonry is the mortar, which traditionally, was mostly clay or lime mixed with sand and silty soil.

Wood is a largely available material in most regions of the world. Since ancient times, it has also been used by humans to build shelters. Even if it is not as durable as masonry and it is combustible, one can find several ancient buildings that use wood in their structures. Many of these structures, particularly when made of hardwood and protected from fire, exhibit remarkable longevity. Still, often wooden construction needs maintenance and allows partial replacement of modules or damaged elements, without compromising the entire structure.

\section{CONCEPTS}

\subsection{Conservation, restoration and rehabilitation}

Conservation is defined in the Nara Charter (ICOMOS, 1994) as "all efforts designed to understand cultural heritage, know its history and meaning, ensure its material safeguard and, as required, its presentation, restoration and enhancement”. A more technical oriented definition can be: all actions or processes that are aimed at safeguarding the character-defining elements of a cultural resource so as to retain its heritage value and extend its physical life.

A different concept is restoration, an action or process of accurately revealing, recovering, or representing the state of a cultural resource or of an individual component, as it appeared at a particular period in its history, while protecting its heritage value. Restoration is a complex concept for the built heritage, as this heritage was hardly produced in any given period of time.

Rehabilitation is often defined as an action or process of making possible a continuing or compatible contemporary use of a cultural resource or an individual component, through repair, alterations, and/or additions, while protecting its heritage value. The problem with this definition is that making possible a modern use according to current standards and codes may be incompatible with sound protection of heritage value.

\subsection{Stabilization, repair and strengthening}

Other relevant technical concepts are stabilization, an action aimed at stopping a deteriorating process involving structural damage or material decay (also applied to actions meant to prevent the partial or total collapse of a deteriorated structure), repair, an action to recover the initial mechanical or strength properties of a material, structural component or structural system (also applied to cases where a structure has experienced a deterioration process having produced a partial loss of its initial performance level), and strengthening, an action providing additional strength to the structure (needed to resist new loading conditions and uses, to comply with a more demanding level of structural safety, or to respond to increasing damage associated with continuous or long term processes).

In the context of conservation of historical structures, repair is not meant to correct any historical deterioration or transformation that only affects the appearance or formal integrity of the building and does not compromise its stability. Repair should be only used to improve structures having experienced severe damage actually conveying a loss of structural performance and thus causing a structural insufficiency with respect to either frequent or exceptional actions. Strict conservation will normally require stabilization or repair operations. Conversely, reha- 
bilitation will frequently lead to strengthening operations.

\section{THE ISCARSAH RECOMMENDATIONS}

\subsection{Basis}

The first conservation attempts resulted often in significant negative experience accumulated, such as blind confidence in modern materials and technologies, mistrust towards traditional materials and original structural resources, devaluation of ancient structural features, and insufficient importance attributed to diagnostic studies before an intervention. On the contrary, modern conservation respects authenticity of the ancient materials and building structure, meaning that interventions must be based on understanding the nature of the structure and the real causes of damage or alterations. Interventions are kept minimal, using an incremental approach, and much importance is attributed to diagnosis studies comprising historical, material and structural aspects.

ICOMOS, the International Council on Monuments and Sites, is a global non-governmental organization, founded in 1965, dedicated to promoting the application of theory, methodology, and scientific techniques to the conservation of the architectural and archaeological heritage. ICOMOS shelters national committees of more than 100 countries and more than 25 international scientific committees.

ISCARSAH is the International Scientific Committee on the Analysis and Restoration of Structures of Architectural Heritage. Founded by ICOMOS in 1996, it is a forum for engineers involved in the restoration and care of building heritage. These aspects were condensed in a document (ICOMOS, 2003), that recognizes that conventional techniques and legal codes or standards oriented to the design of new buildings may be difficult to apply, or even inapplicable, to heritage buildings, and stating the importance of a scientific and multidisciplinary approach involving historical investigation, inspection, monitoring and structural analysis.

\subsection{Principles and guidelines}

A multi-disciplinary approach is obviously required in any conservation or rehabilitation project and the peculiarity of cultural heritage buildings, with their complex history, requires the organization of studies and analysis in steps that are similar to those used in medicine. Anamnesis, diagnosis, therapy and controls, corresponding respectively to the condition survey, identification of the causes of damage and decay, choice of the remedial measures and control of the efficiency of the interventions.

The phases of the study involve:

- Diagnosis: Identification of the causes of damage and decay;
- Safety evaluation: Definition of the acceptability of safety levels by analyzing present condition of structure and materials;

- Design of remedial measures: Layout of repair or strengthening actions to ascertain the required safety.

Diagnosis and safety evaluation of the structure are two consecutive and related stages on the basis of which the effective need for and extent of treatment measures are determined. If these stages are performed incorrectly, the resulting decisions will be arbitrary: poor judgement may result in either conservative and therefore heavy-handed conservation measures or inadequate safety levels.

All phases should be based on both qualitative (such as historical investigation and inspection) and quantitative (such as monitoring and structural analysis) methods that take into account the effect of the phenomena on structural behavior. It is stressed that the approach adopts a scientific method to reach conclusions on the condition of the building and optimal interventions, resorting to sources such as historical information, inspection of current condition or monitoring, which provide empirical data, and structural modeling, which is based on a hypothetical representation of the reality. Certainly, that models are a very important contribution, even if they will not represent the full reality and must be validated, while their possibilities are always limited to some extent. In a first step, the models are calibrated and validated against in situ testing or performance, while, in a second step, they are used for extrapolating the behavior and for defining the safety level.

Still, there are several difficulties, namely with respect to the limited applicability of available codes and subjectivity. Codes prepared for the design of modern structures are often inappropriately applied to historic structures. They are based in calculation approaches that may fail to recognize the real structural behavior and safety condition of ancient constructions. The enforcement of seismic and geotechnical codes, can lead to drastic and often unnecessary measures that fail to take into account the real structural behavior. Nevertheless, recent standardization advances have been made, e.g. in Italy (PCM, 2007) and USA (ASCE, 2013).

In addition, any assessment of safety is affected by two types of uncertainties. First, the uncertainty attached to data used (actions, geometry, deformations, material properties...). Second, the difficulty of representing real phenomena in a precise way with an adequate mathematical model. The subjective aspects involved in the study and evaluation of a historic building may lead to conclusions of uncertain reliability.

Modern legal codes and professional codes of practice adopt a conservative approach involving the application of safety factors to take into account the 
various uncertainties. This is appropriate for new structures where safety can be increased with a small increase in member size and cost. However, such an approach might not be appropriate in historic structures where requirements to improve the capacity may lead to the loss of historic fabric or to changes in the original conception of the structure. A more flexible and broader approach, needs to be adopted for historic structures to relate the remedial measures more clearly to the actual structural behavior and to retain the principle of minimum intervention, limiting in any case risk to an acceptable level.

\section{METHODOLOGICAL ASPECTS}

\subsection{Diagnosis}

Many developments have been recently made, namely on investigation procedures for the diagnosis of historic fabric, e.g. Binda et al. (2000) and Kasal \& Anthony (2004). Visual inspection is one of the most important tasks to be carried out for structural diagnosis, requiring adequate training and expertise. This often requires opening up the structure, if possible, and the use of additional equipment, such as a boroscopic camera (for internal vision), a laser scan or a total station (for geometry and deformation definition), among others.

Several non-destructive tests (NDT) can be used for the experimental determination of the mechanical, physical or chemical properties of materials or structural members. These tests do not cause any loss of, or damage to, the historic fabric and, therefore, sometimes the synonymous term noninvasive techniques is used, see Fig. 2 for examples. These can be based on elastic waves (e.g. ultrasonic and sonic testing), in electromagnetic waves (e.g. ground probe radar) and in other concepts. Alternatively, minor destructive testing (MDT) causes minimal and easily reparable damage to the historic fabric. Among many examples, coring, flat-jack testing or drilling resistance are popular techniques.

\subsection{Safety evaluation}

Many methods and simulation tools available for the assessment of the safety of historic masonry structures. The methods have different levels of complexity (from simple graphical methods and hand calculations to complex mathematical formulations and large systems of equations), different availability for the practitioner (from well disseminated structural analysis tools accessible to any consulting engineer office to advanced structural analysis tools only available in a few research oriented institutions and large consulting offices), different time requirements (from a few seconds of computer time to a number of days of processing) and, of course, different costs. Still, many structural analysis techniques can be adequate, possibly for different applications, if com- bined with proper engineering reasoning, see Lourenço (2002) for a review.

Seismic assessment of historic built heritage is rather complex, as the safety assessment techniques used for modern buildings usually fail to accurately replicate the true behavior of such structures. Many advances have been made in the last decades, namely with respect to macro-block and macro-element analysis, see Lourenço et al. (2011) and Fig. 3.

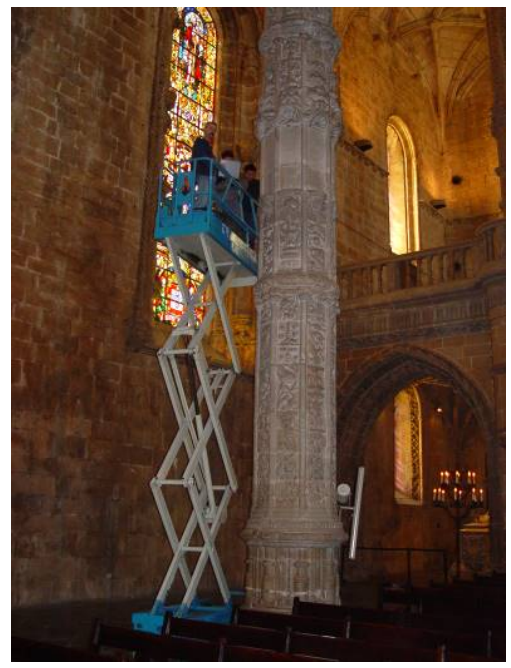

(a)

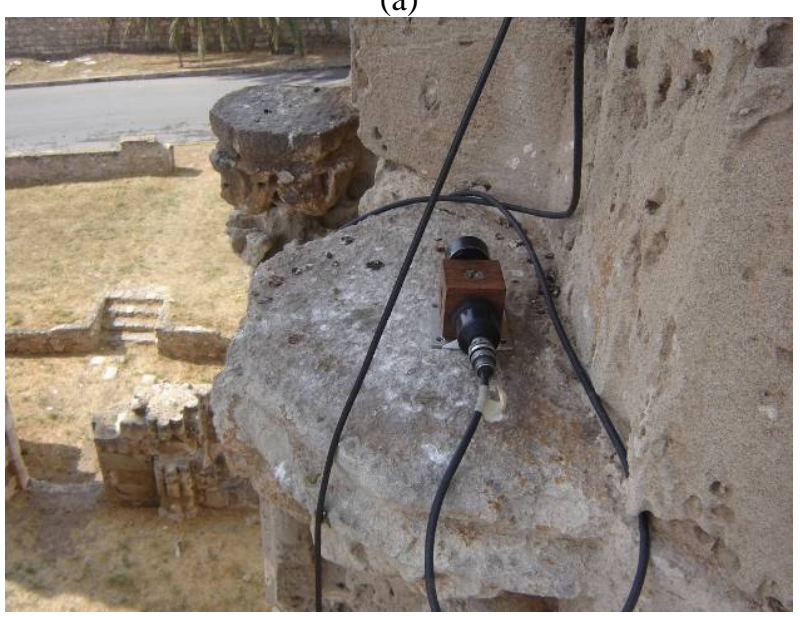

(b)

Figure 2.: Non-destructive testing: (a) Ground Probe Radar testing at Monastery of Jerónimos, Lisbon, Portugal; (b) Dynamic identification at Famagusta, Cyprus.

Masonry is a heterogeneous material that consists of units and joints. Usually, joints are weak planes and concentrate most damage in tension and shear. The use of modern structural analysis techniques requires a thorough experimental description of the existing materials. This information is available in great extent, given the recent investment in studying existing materials. In particular, the Italian normative provides a wealth of information regarding aspects such as compressive strength, shear strength, Young's modulus, shear modulus and specific weight (PCM, 2003). In addition, several aspects can be taken into account, such as the quality of the mortar, the thickness of the joints, the presence of regular courses of masonry, the regular presence of through stones, the presence of an internal infill lay- 
er, the strengthening with grout injection or the strengthening with reinforced plaster.

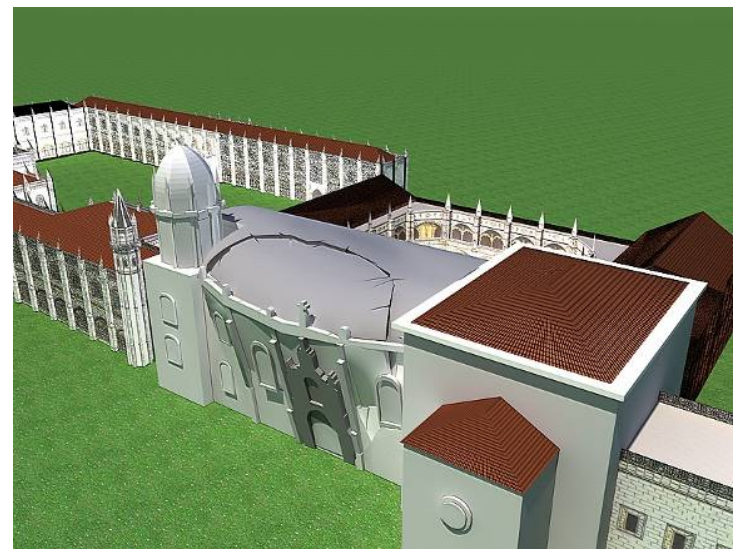

(a)
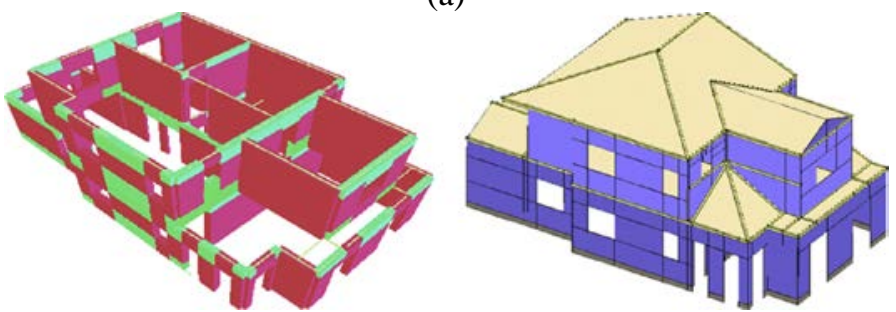

(b)

Figure 3: Advanced numerical simulations for seismic safety assessment, using: (a) Finite elements for Monastery of Jerónimos, Lisbon, Portugal; (b) Macro-elements for residential masonry structures.

\subsection{Remedial measures}

Any remedial actions should respect existing materials and structure, and are expected to have minimal impact. The basis for design include safety, compatibility, least invasivity, durability, reversibility and controllability. Injection grouts, for example, are a much used remedial technique, which can be durable and mechanically efficient while preserving historic values. Still, the selection of a grout for repair must be based on the physical and chemical properties of the existing materials. Parameters such as rheology, injectability, stability, and bond of the mix should be considered to ensure the effectiveness of grout injection. The insertion of bars (ideally stainless steel or composite) within the masonry using coring also has been a popular technique to enhance structural capacity.

Increasing consideration has been given in recent years to the development of innovative technologies that apply externally bonded reinforcement systems using composite materials for strengthen, see Fig. 4a,b ing. Applications of fiber reinforced polymers (FRP) to vaults, columns, and walls have demonstrated their effectiveness in increasing loadcarrying capacity and in upgrading seismic strength, even if concerns on durability exist. During the past decade, in an effort to alleviate some drawbacks associated with the use of polymer-based composites, inorganic matrix composites have been developed. This broad category includes steel reinforced grouts
(SRG, unidirectional steel cords embedded in a cement or lime grout), fabric-reinforced cementitious matrix (FRCM) composites / textile reinforced mortars (TRM) (a sequence of one or more layers of cement-based matrix reinforced with dry fibers in the form of open single or multiple meshes). Currently, natural fibers are becoming increasing popular as a green research field for crack control and strengthening, see Fig. 4c.

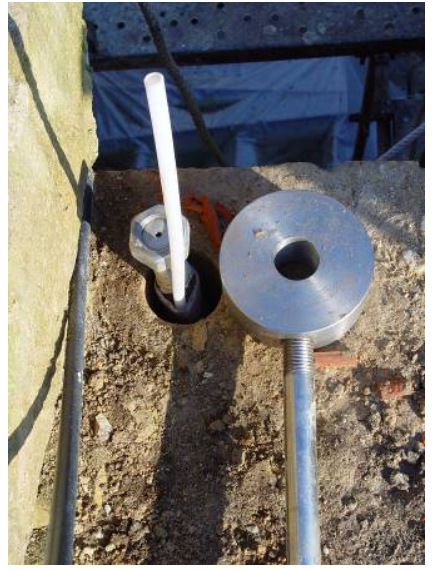

(a)

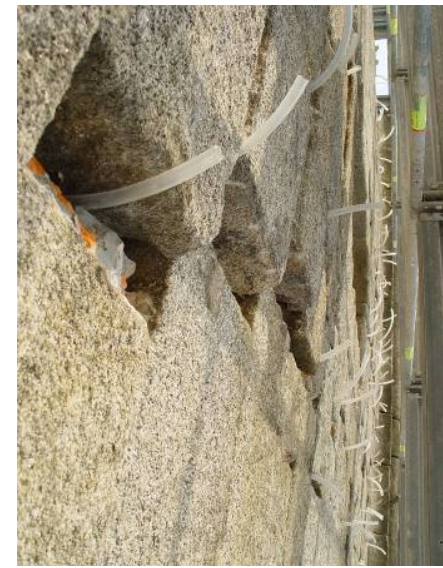

(b)

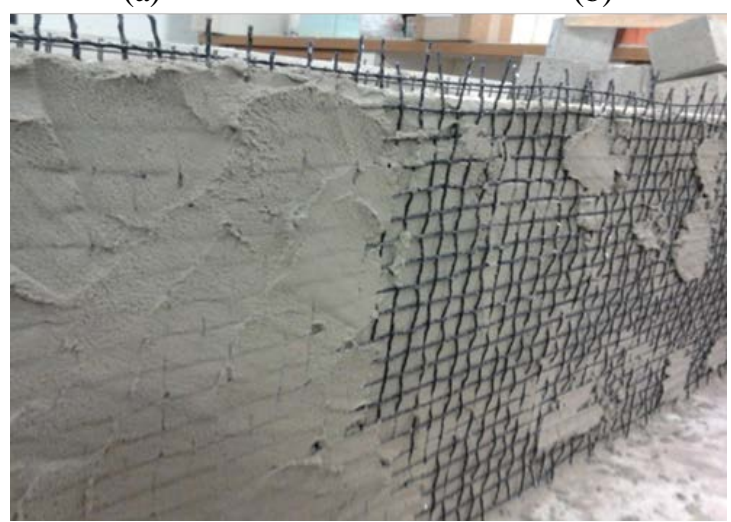

(c)

Figure 4: Examples of application of remedial measures: (a) insertion of stainless steel bars; (b) injection of lime based grout; (c) textile reinforced mortars.

\section{CONCLUSIONS}

Earthquakes are, and will remain, one of the most powerful sources of destruction for cultural heritage buildings. Many developments have recently been made, namely on methodological aspects, investigation procedures for the diagnosis of historic fabric, structural analysis techniques or remedial measures. The application of these developments to vernacular construction is possible and needed, to retain its heritage value and to reach cost-effective interventions.

Cracking occurs at early stages of loading, and adequate approaches for safety assessment are available, together with a wealth of information on mechanical characterization. Recent developments in intervention techniques that better confine and tie together building parts, thereby reducing the possibility of separation of parts and disintegration of individual elements during a seismic event, are significant. 


\section{ACKNOWLEDGEMENTS}

The authors gratefully acknowledge the partial support by the research project 'SEISMIC-V - Vernacular Seismic Culture in Portugal' (PTDC/ATPAQI/ 3934/2012), from the Portuguese Science and Technology Foundation (FCT).

\section{REFERENCES}

ASCE, 2013. Seismic Evaluation and Retrofit of Existing Buildings. ASCE Standard ASCE/SEI 41-13.

Binda, L., Saisi, A. \& Tiraboschi, C. 2000. Investigation procedures for the diagnosis of historic masonries. Construction and Building Materials 14(4): 199-233.

ICOMOS, 1994. The Nara Document on Authenticity, International Council on Monuments and Sites.

ICOMOS, 2003. Recommendations for the Analysis and Restoration of Historical Structures, ISCARSAH, International Council on Monuments and Sites.

Kasal, B. \& Anthony, R.W. 2004. Advances in in situ evaluation of timber structures. Progress in Structural Engineering and Materials 6(2): 94-103.

Leite, J., Lourenço, P.B. \& Ingham, J.M. 2013. Statistical Assessment of Damage to Churches Affected by the 20102011 Canterbury (New Zealand) Earthquake Sequence. Journal of Earthquake Engineering 17(1): 73-97.

Lourenço, P.B. 2002. Computations of historical masonry constructions Progress in Structural Engineering and Materials 4(3): 301-319.

Lourenço, P.B., Mendes, N., Ramos, L.F. \& Oliveira, D.V. 2011. Analysis of masonry structures without box behavior, International Journal of Architectural Heritage 5(4-5): 369-382.

Neves, F., Costa, A., Vicente, R., Oliveira, C.S. \& Varum, H. 2012. Seismic vulnerability assessment and characterisation of the buildings on Faial Island, Azores. Bulletin of Earthquake Engineering 10 (1): 27-44.

PCM, 2003. Technical standards for seismic design of structures. Directive of the Prime Minister, 20/03/2003. G.U. n.252 of 29/10/2003. Modified by OPCM 3431, 3/5/2005.

PCM, 2007. Guidelines for evaluation and mitigation of seismic risk to cultural heritage. Directive of the Prime Minister, 12/10/2007. G.U. n.24 of 29/01/2008. Gangemi Editor. Rome. ISBN 978-88-492-1269-3. 